\title{
Improvements in Low Voltage PFIB for Sample Preparation and Large Volume Serial Sectioning Tomography
}

Brandon van Leer ${ }^{1}$, Lee Casalena ${ }^{1}$, Jeremy Graham ${ }^{1}$ and Bartlomiej Winiarski ${ }^{2}$

${ }^{1}$ ThermoFisher Scientific, Hillsboro, Oregon, United States, ${ }^{2}$ ThermoFisher Scientific /The University of Manchester, Brno, Jihomoravsky kraj, Czech Republic

Compared to $\mathrm{Ga}^{+}$liquid metal ion source (LMIS) DualBeam FIB-SEMs, inductively coupled plasma (ICP) source DualBeams are relatively new [1]. Until recently, only $\mathrm{Ga}^{+}$and $\mathrm{Xe}^{+}$source technologies have been commercially available. State-of-the-art DualBeam PFIB-SEMs allow fast switching between ion sources of xenon, argon, oxygen and nitrogen. 30 years ago, the first S/TEM sample was prepared using a $\mathrm{Ga}^{+}$FIB. During those 30 years, many advances in low energy FIB performance for site-specific FIB sample preparation have occurred to improve sample quality. Modern HR-STEM and HR-TEM systems require very thin specimens with minimal surface damage for the best results. Minimizing surface damage during FIB specimen preparation is an important factor for high quality images and analytical results. Using conventional $\mathrm{Ga}^{+} \mathrm{FIB}$ milling, techniques using reduced accelerating voltages for final polishing to minimize sample damage are commonly employed in PFIB DualBeams [2]. Nowadays, new column and source technologies allow for multiple ion species to be employed for sputtering at high and low accelerating voltages without any hardware changes. Previous PFIB column technologies only allowed for low energy polishing down to $2 \mathrm{kV}$. New advances in hardware technology and optimization of column parameters now allow users to utilize accelerating voltages down to $500 \mathrm{~V}$. With the introduction of ultra-low voltage milling along with alternative ion species $\left(\mathrm{Xe}^{+}, \mathrm{Ar}^{+}, \mathrm{O}^{+}, \mathrm{N}^{+}\right)$, it is of interest to characterize the ion-solid interaction with respect to FIB sidewall damage in an effort to understand whether ion species other than $\mathrm{Ga}^{+}$might produce better TEM samples for high resolution STEM (HR-STEM) imaging.

The use of low voltage PFIB combined with spin milling for serial sectioning tomography (SST) (introduced by Winiarski et al) offers new capabilities for researchers interested in surface quality enhancements afforded by low voltage FIB polishing [3]. It is well known that low voltage FIB polishing improves surface quality (leading to higher indexing rates) in metals for EBSD analysis, but can require significantly more processing time [4]. The combination of spin mill with low voltage PFIB is an application that can allow for large area, HR-EBSD analysis and even large volume SST with a high index rate.

In this work, we present FIB sidewall damage results in silicon using $\mathrm{Xe}^{+}$and $\mathrm{Ar}^{+}$ions at $1 \mathrm{kV}$ and $500 \mathrm{~V}$ for S/TEM sample preparation and examine low energy PFIB milling at small glancing angles $(<2$ degrees) to improve surface quality across large areas for analytical techniques like EBSD. 


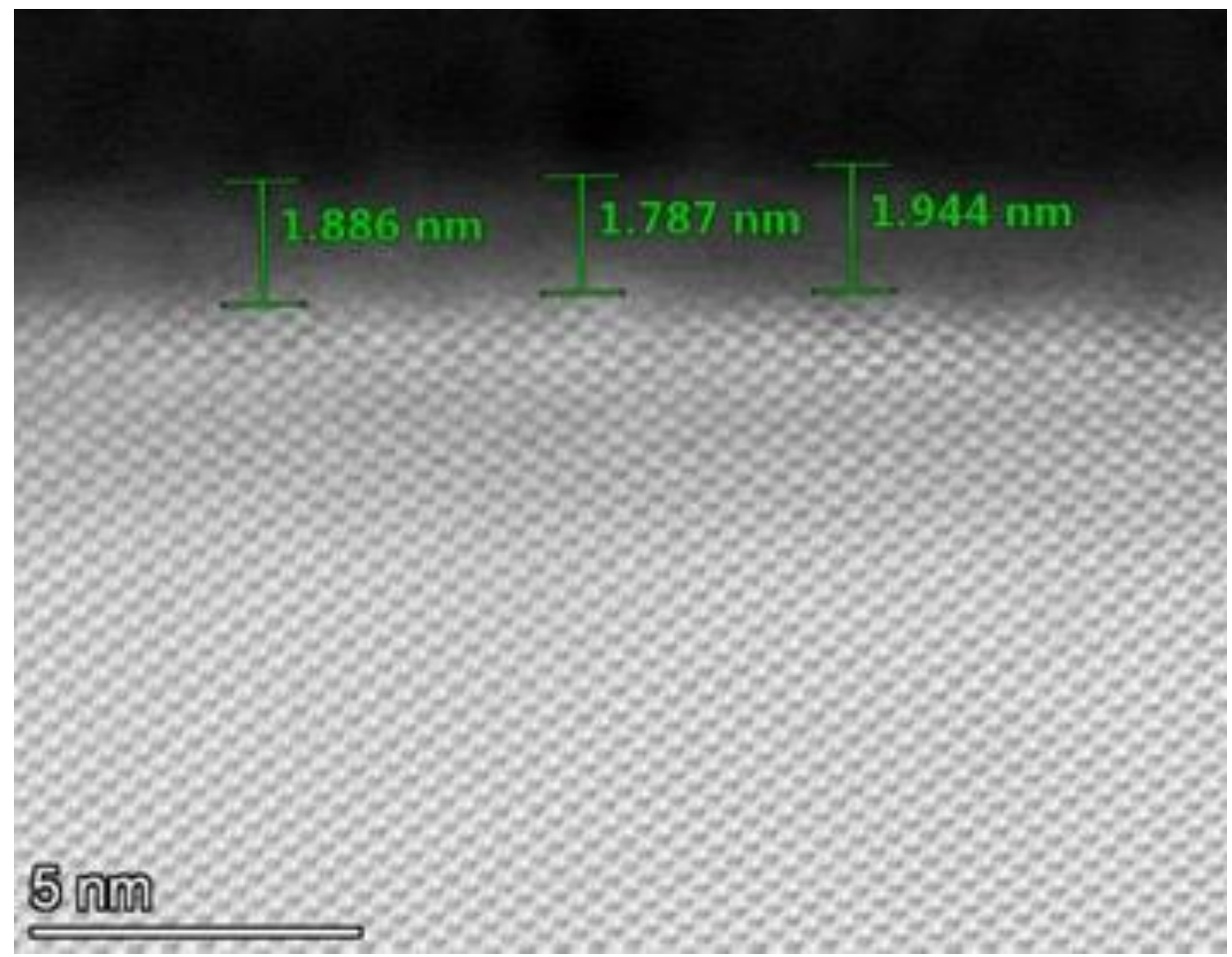

Figure 1. $300 \mathrm{keV}$ HR-STEM image of amorphous sidewall damage in Si from an Xe+ PFIB with $1 \mathrm{kV}$.

FIB Sidewall Damage, $\mathrm{nm}$

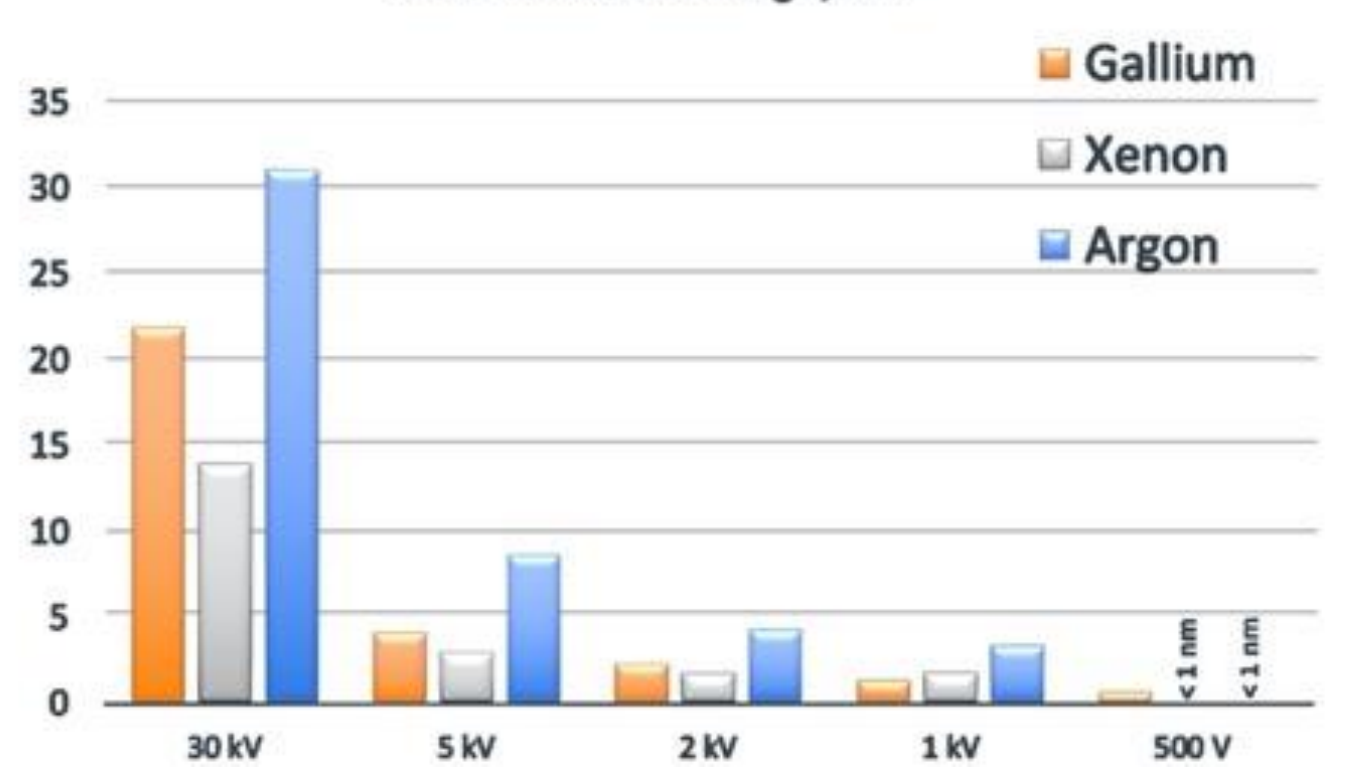

Figure 2. Chart of FIB sidewall damage in $\mathrm{Si}$ for $\mathrm{Ga}+\mathrm{Xe}+$ and $\mathrm{Ar}+$ as a function of accelerating voltage.

References

[1] P. Tesch et al, Proceedings from the $34^{\text {th }}$ Inter. Symposium for Testing and Failure Analysis (2008).

[2] B. Van Leer et al, Microscopy and Microanalysis 25 (Suppl 2), (2019).

[3] B. Winiarski et al, Microscopy and Microanalysis 25 (Suppl 2), (2019).

[4] J. Michael and P. Kotula, Microscopy and Microanalysis, 14 (Suppl 2), (2008). 